it, or including that which we, $2 c$ cycles before, deleted from it, until the $\left(2^{k-1}\right)$ st combination, which corresponds to the empty set plus element $k$.

Proof of (1). Since the binary representation of $2^{k-1}$ is a 1 bit followed by $(k-1)$ zeros, the $k$ th element is included on cycle $2^{k-1}$. The $k$ th element will remain until the binary number 11 followed by $(k-1)$ zeros appears. This will be on cycle number $\left(2^{k}+2^{k-1}\right)>\left(2^{k}-1\right)$. Thus, all combinations from $2^{k-1}$ through $\left(2^{k}-1\right)$ will include the $k$ th element.

Proof of (2). Since $\left(2^{k-1}+c\right)+\left(2^{k-1}-c\right)=2^{k}$, the binary representations of $\left(2^{k-1}+c\right)$ and $\left(2^{k-1}-c\right)$ correspond in all their low-order zeros, and the low-order 1 , in which they also correspond. The bit above the 1 must differ in the two numbers, due to the binary carry. Thus, $B\left(2^{k-1}+c\right)=-B\left(2^{k-1}-c\right)$.

To complete the proof by induction, we may note, by Table 1 , that the algorithm has generated all combinations for $k \leqq 4$.

University of Pittsburgh, and American Institute for Research Pittsburgh, $\mathrm{Pa}$.

\title{
Generation of Permutations by Addition
}

\section{By John R. Howell}

1. Introduction. Suppose one wishes to generate the $k$ ! permutations of $k$ distinct marks. Representing these $k$ marks by $0,1,2, \cdots,(k-1)$ written side by side to form the "digits" of a base $k$ integer, then the repeated addition of 1 will generate integers whose "digits" represent permutations of $k$ marks. Many numbers are also generated which are not permutations. D. H. Lehmer [2] states that this so-called addition method can be made more efficient by adding more than 1 to each successive integer.

2. Method. In this note, we show that the correct number greater than 1 to add to this integer is a multiple of $(k-1)$ radix $k$.

LEMMA 1. The arithmetic difference radix $k$ between an integer composed of mutually unlike digits and another integer composed of a permutation of the same digits is a multiple of $(k-1)$.

Considering the process of "casting out nines," it is obvious that the two integers are congruent mod $(k-1)$. Hence, their difference is zero $\bmod (k-1)$.

The method seems to have two advantages. First, one can generate all $k$ ! permutations in lexicographic order. Second, all permutations "between" two given permutations can be obtained. The process can be made to be cyclic if upon obtaining $(k-1), \cdots, 0$ one takes the next permutation to be $0,1, \cdots,(k-1)$.

3. Example. Suppose we wish to generate the 4 ! permutations of 4 marks. Representing these 4 marks by $0,1,2$ and 3, we add 3 radix 4 to 0123 to get 0132 . Continuing this process we get the 4 ! permutations desired. The array below shows

Received September 11, 1961. 
the first 16 numbers generated by this process. An asterisk marks each integer whose digits represent a required permutation. The other integers were rejected because of the occurrence of repeated digits.

$\begin{array}{clcc}\text { Sequence } & \text { Integer } & \text { Sequence } & \text { Integer } \\ 1 & 0123^{*} & 9 & 0303 \\ 2 & 0132^{*} & 10 & 0312^{*} \\ 3 & 0201 & 11 & 0321^{*} \\ 4 & 0210 & 12 & 0330 \\ 5 & 0213^{*} & 13 & 0333 \\ 6 & 0222 & 14 & 1002 \\ 7 & 0231^{*} & 15 & 1011 \\ 8 & 0300 & 16 & 1020\end{array}$

4. Adaptation to a Computer. In a computer such as the IBM 7090 where convert instructions are available it is easy to do radix $k$ arithmetic. Otherwise one could simulate the process by adding 9 digit-wise and testing the resulting sum for having unique digits each one of which is one of the original $k$ digits.

5. Acknowledgments. This method was developed when the author was with the Statistics Department, Agricultural Experiment Station, University of Florida, Gainesville, Florida, in connection with the problem of obtaining a particular arrangement of the rows of a Latin square. He wishes to thank Mark Robinson of Martin Marietta Corp. for suggestions concerning the writing of the manuscript.

Computer Applications Department

Martin Marietta Corporation

Orlando, Florida

1. C. B. Tomprins, "Machine attacks on problems whose variables are permutations," Proceedings of Symposia in Applied Mathematics, v. VI, Numerical Analysis, McGraw-Hill, New York, 1956, p. 195-211.

2. D. H. LEHMER, "Teaching combinatorial tricks to a computer," Proceedings of Symposia in Applied Mathematics, v. X, Combinatorial Analysis, American Mathematical Society, Providence, R. I., 1960, p. 179-193.

3. MARK B. Wells, "Generation of permutations by transposition," Math. Comp. v. 15, 1961, p. 192-195.

\section{Multiple Quadrature with Central Differences on One Line}

\section{By Herbert E. Salzer}

Abstract. The coefficients $A_{2 m}^{n}$ in the $n$-fold quadrature formulas for the stepwise integration of $(1) y^{(n)}=f\left(x, y, y^{\prime}, \cdots, y^{(n-1)}\right)$, at intervals of $h$, namely, for $n$ even, (2) $\delta^{n} y_{0}=h^{n} \sum_{m=1}^{10}\left(1+A_{2 m}^{n} \delta^{2 m}\right) f_{0}+\cdots$, for $n$ odd, (3) $\mu \delta^{n} y_{0}=h^{n} \sum_{m=1}^{10}$ $\left(1+A_{2 m}^{n} \delta^{2 m}\right) f_{0}+\cdots$, are tabulated exactly for $n=1(1) 6, m=1(1) 10$. They were calculated from the well-known symbolic formulas (4) $\delta^{n} y=(\delta / D)^{n} f,(5)$ $(\delta / D)^{n}=\left(\delta h / 2 \sinh ^{-1}(\delta / 2)\right)^{n}$ and $(6) \mu=\left(1+\delta^{2} / 4\right)^{1 / 2}=1+\frac{\delta^{2}}{8}-\frac{\delta^{4}}{128}+\frac{\delta^{6}}{1024}-$ 